\title{
ENERGY-PRESERVING RUNGE-KUTTA METHODS
}

\author{
Elena Celledoni ${ }^{1}$, Robert I. McLachlan ${ }^{2}$, David I. McLaren ${ }^{3}$, \\ Brynjulf Owren ${ }^{1}$, G. Reinout W. Quispel ${ }^{3}$ and William M. Wright ${ }^{3}$
}

\begin{abstract}
We show that while Runge-Kutta methods cannot preserve polynomial invariants in general, they can preserve polynomials that are the energy invariant of canonical Hamiltonian systems.
\end{abstract}

Mathematics Subject Classification. 65P10, 65L06.

Received May 21, 2008.

Published online July 8, 2009.

All Runge-Kutta (RK) methods preserve arbitrary linear invariants [12], and some (the symplectic) RK methods preserve arbitrary quadratic invariants [4]. However, no RK method can preserve arbitrary polynomial invariants of degree 3 or higher of arbitrary vector fields [1]; the linear system $\dot{x}=x, \dot{y}=y, \dot{z}=-2 z$, with invariant $x y z$, provides an example. (Preservation would require $R(h)^{2} R(-2 h) \equiv 1$, where $R$ is the stability function of the $\operatorname{method}^{4}$; but this requires $R(h)=\mathrm{e}^{h}$, which is impossible [6].) This result does not rule out, however, the existence of RK methods that preserve particular (as opposed to arbitrary) invariants. Since the invariant does not appear in the RK method, this will require some special relationship between the invariant and the vector field. Such a relationship does exist in the case of the energy invariant of canonical Hamiltonian systems: see [3,5] on energy-preserving B-series. In this article we show that for any polynomial Hamiltonian function, there exists an RK method of any order that preserves it.

The key is the average vector field (AVF) method first written down in [9] and identified as energy-preserving and as a B-series method in [10]: for the differential equation

$$
\dot{x}=f(x), \quad x \in \mathbb{R}^{n}
$$

the AVF method is the map $x \mapsto x^{\prime}$ defined by

$$
\frac{x^{\prime}-x}{h}=\int_{0}^{1} f\left(\xi x^{\prime}+(1-\xi) x\right) \mathrm{d} \xi .
$$

Keywords and phrases. B-series, Hamiltonian systems, energy-preserving integrators, Runge-Kutta methods.

1 Department of Mathematical Sciences, NTNU, 7491 Trondheim, Norway.

2 Institute of Fundamental Sciences, Massey University, Palmerston North, New Zealand. r.mclachlan@massey.ac.nz

3 Mathematics Department, La Trobe University, VIC 3086, Australia.

${ }^{4}$ That is, when applied to the scalar ODE $\dot{x}=\lambda x$ the integrator yields the map $x \mapsto R(h \lambda) x$. 
Proposition 1. The AVF method is a B-series method, is affine-covariant $t^{5}$, self-adjoint $t^{5}$, and of order 2. When

$$
f(x)=J^{-1} \nabla H(x), \quad J=\left(\begin{array}{cc}
0 & -I \\
I & 0
\end{array}\right), H: \mathbb{R}^{n} \rightarrow \mathbb{R},
$$

the AVF method preserves the energy H. It is pseudo-symplectic of order 2 (but not 3) and conjugate-pseudosymplectic of order 4 (but not 5).

Proof. The existence of the B-series can be seen simply by expanding $x^{\prime}$ in a Taylor series. Alternatively, the B-series can be calculated by substituting the assumed B-series ${ }^{6}$

$$
x^{\prime}=x+\sum_{t \in T} \frac{h^{|t|}}{\sigma(t)} b(t) F(t)
$$

for $x^{\prime}$ into $f\left(\xi x^{\prime}+(1-\xi) x\right)$, evaluating the coefficients of the latter using Lemma III.1.9 of [6], and integrating term-by-term, to get

That is,

$$
b(\bullet)=1, \quad b\left(\left[t_{1}, \ldots, t_{n}\right]\right)=\frac{1}{n+1} b\left(t_{1}\right) \ldots b\left(t_{n}\right) .
$$

$$
x^{\prime}=x+h f+\frac{1}{2} h^{2} f^{\prime}(f)+h^{3}\left(\frac{1}{6} f^{\prime \prime}(f, f)+\frac{1}{4} f^{\prime}\left(f^{\prime}(f)\right)\right)+\ldots
$$

which can be compared to the expansion of the exact solution,

$$
x(h)=x+h f+\frac{1}{2} h^{2} f^{\prime}(f)+h^{3}\left(\frac{1}{6} f^{\prime \prime}(f, f)+\frac{1}{6} f^{\prime}\left(f^{\prime}(f)\right)\right)+\ldots,
$$

showing that the order is 2. All B-series methods are affine-covariant. Self-adjointness is easily checked using the change of variables $\xi^{\prime}=1-\xi$ in (2). The change in energy in one step is (where ()$^{\top}$ denotes transpose)

$$
\begin{aligned}
H\left(x^{\prime}\right)-H(x) & =\int_{0}^{1} \nabla H\left(\xi x^{\prime}+(1-\xi) x\right)^{\top}\left(x^{\prime}-x\right) \mathrm{d} \xi \\
& =h\left(\int_{0}^{1} \nabla H\left(\xi x^{\prime}+(1-\xi) x\right) \mathrm{d} \xi\right)^{\top}\left(\int_{0}^{1} f\left(\xi x^{\prime}+(1-\xi) x\right) \mathrm{d} \xi\right) \\
& =h\left(\int_{0}^{1} \nabla H\left(\xi x^{\prime}+(1-\xi) x\right) \mathrm{d} \xi\right)^{\top} J^{-1}\left(\int_{0}^{1} \nabla H\left(\xi x^{\prime}+(1-\xi) x\right) \mathrm{d} \xi\right) \\
& =0 .
\end{aligned}
$$

Because the AVF method has order 2, it is pseudo-symplectic of order 2 (see [6] for an introduction to pseudosymplecticity). The condition to be pseudo-symplectic of order 3 is $b(u \circ v)+b(v \circ u)-b(u) b(v)=0$ for $u=\circ$ and

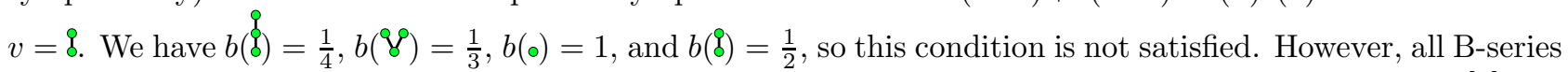
methods of order 2 are conjugate-pseudo-symplectic of order 3 in general and 4 if they are self-adjoint [6]. To check conjugate-pseudo-symplecticity at order 5 we calculate all 9 coefficients of the B-series at order 5 and

\footnotetext{
${ }^{5}$ The integrator $x \mapsto x^{\prime}=\varphi_{h, f}(x)$ for the vector field $f$ is said to be covariant with respect to the map $x \mapsto S(x)$ if $S \circ \varphi_{h, f} \circ S^{-1}=$ $\varphi_{h,(D S . f) \circ S^{-1}}$, i.e., if the operations of applying $S$ and the integrator commute. It is affine-covariant if it is covariant with respect to all affine maps $x \mapsto A x+b$. It is said to be self-adjoint (or symmetric) if $\varphi_{h}=\varphi_{-h}^{-1}$; if $f$ has $S$ as a reversing symmetry, then $S$-covariance and self-adjointness ensure that $\varphi_{h, f}$ is reversible [8].

${ }^{6}$ See [6] for background on B-series. Here $T$ is the set of rooted trees, $F(t)$ is the elementary differential associated with the tree $t,|t|$ is the number of vertices of $t$, and $\sigma(t)$ is the symmetry coefficient (number of automorphisms) of $t$. For example, Y $\in T$, $\sigma(\mathcal{Y})=2$, and $F(\mathcal{Y})=f^{\prime \prime}(f, f)$.
} 
check the 3 conditions given as equations (3.7.18)-(3.18.20) of Scully [11] and too lengthy to reproduce here; they are not satisfied.

While the AVF method requires, in general, the evaluation of the integrals of functions of one variable, the following proposition can often be used to avoid this. By linearity, it extends to give the AVF for Hamiltonians that are sums of functions of 1 variable.

Proposition 2. If $H=H\left(a^{\top} x\right)$ then

$$
\int_{0}^{1} f\left(\xi x^{\prime}+(1-\xi) x\right) \mathrm{d} \xi=J^{-1} a \frac{H\left(a^{\top} x^{\prime}\right)-H\left(a^{\top} x\right)}{a^{\top} x^{\prime}-a^{\top} x} .
$$

Proof. We have $f=J^{-1} a H^{\prime}\left(a^{\top} x\right)$, and $\int_{0}^{1} H^{\prime}\left(a^{\top} x^{\prime} \xi+a^{\top} x(1-\xi)\right) \mathrm{d} \xi$ can be integrated directly to give the result.

We now replace the integral in (2) by a quadrature to get the following.

Proposition 3. Let $\left(b_{i}, c_{i}\right), i=1, \ldots, s$ be the weights and nodes of a quadrature rule on $[0,1]$ that is exact on polynomials of degree $m-1$. Let $H$ be a polynomial of degree $m$. Then the s-stage Runge-Kutta method

$$
\frac{x^{\prime}-x}{h}=\sum_{i=1}^{s} b_{i} f\left(x+\left(x^{\prime}-x\right) c_{i}\right)
$$

preserves $H$, is consistent, mono-implicit, of order $\min (m, 2)$, is self-adjoint when the quadrature rule is symmetric, and has Butcher tableau $A=c b^{\top}$.

Gauss quadrature, for example, preserves all polynomial Hamiltonians up to degree $2 s$. The B-series of methods of the form (10) have the same coefficients as the AVF method (i.e., (5)) for trees with at most $2 s-1$ branches at each vertex. Compositions of (10) and its adjoint then give RK methods of any order that preserve the energy ${ }^{7}$.

Some examples are the midpoint rule (energy-preserving for quadratic Hamiltonians) and Simpson's rule

$$
\frac{x^{\prime}-x}{h}=\frac{1}{6} f(x)+\frac{2}{3} f\left(\frac{x+x^{\prime}}{2}\right)+\frac{1}{6} f\left(x^{\prime}\right)
$$

that is second-order, self-adjoint, and energy-preserving for quartic Hamiltonians. One can also regard the representation (10) as a good way to implement (2) when $H$ is a polynomial.

Such an existence result motivates the study of energy-preserving RK methods in general.

Proposition 4. An energy-preserving B-series must satisfy all the quadrature order conditions. No RK method is energy-preserving in general.

\footnotetext{
${ }^{7}$ Proposition 3 contradicts non-existence results in [7], namely Propositions 1 (a necessary condition for an RK method to preserve a generic invariant is that it be symplectic; the methods (10) are not symplectic), 3 (a necessary condition for an RK method to preserve an invariant is that the invariant satisfy a certain PDE), and 5 (an RK method cannot preserve any given polynomial invariant) of [7]. These propositions are false. The error in the proofs can be seen by considering the final equation in the proof of Proposition 1 of [7]: in our notation,

$$
H\left(x^{\prime}\right)-H(x)=\frac{1}{2} h^{2} \sum_{i, j=1}^{s} M_{i j} \mathcal{B}(H)\left(X_{i}\right)+\mathcal{O}\left(h^{3}\right), \quad M_{i j}=b_{i} b_{j}-b_{i} a_{i j}-b_{j} a_{j i}
$$

where the $X_{i}$ are the stages of the RK method and $\mathcal{B}(H)$ is a certain differential polynomial in $H$, from which it is concluded that, unless $\mathcal{B}(H) \equiv 0$, for annihilation of the $\mathcal{O}\left(h^{2}\right)$ terms it is necessary that $M_{i j}=0$ for all $i$ and $j$. In fact this is only sufficient. The $\mathcal{O}\left(h^{2}\right)$ terms are not independent; one can substitute $X_{i}=x+h \sum_{j=1}^{s} a_{i j} f\left(X_{j}\right)$, then at $\mathcal{O}\left(h^{2}\right)$ all one gets is the necessary condition $\sum_{i, j=1}^{s} M_{i j}=0$, which, on rearranging, is the condition that the method be of order 2 .
} 
Proof. Consider the Hamiltonian $H=p-F(q)$ with ODEs $\dot{q}=1, \dot{p}=f(q)$ where $F^{\prime}=f$. The elementary differential associated with the bushy tree with $n$ leaves is $\left(\delta_{n 0}, f^{(n)}(q)\right)$. All B-series methods give $q^{\prime}=q+h$. Energy preservation requires $p^{\prime}-F\left(q^{\prime}\right)=p-F(q)$ or $p^{\prime}=p+\int_{t_{0}}^{t_{0}+h} f(t) \mathrm{d} t=p+\sum_{n=1}^{\infty} \frac{h^{n}}{n !} f^{(n-1)}\left(t_{0}\right)$; the terms of this Taylor series give the quadrature order conditions on the bushy trees.

Applying an RK method to this system gives $p^{\prime}=p+h \sum_{i=1}^{s} b_{i} f\left(t_{0}+c_{i} h\right)$. This cannot equal $p+\int_{t_{0}}^{t_{0}+h} f(t) \mathrm{d} t$ for all $f$ because the quadrature rule cannot be exact on all $f$. (Choose an $f$ that is zero at the quadrature nodes but has nonzero integral.)

The same proof shows that no B-series method that uses only a finite number of values of $f$ and its derivatives can be energy-preserving in general. Some kind of integral, as in the AVF method (2), is required.

Proposition 5. A Runge-Kutta method with stability function $R(z)$ is energy-preserving for all quadratic Hamiltonians iff $R(z) R(-z) \equiv 1$.

Proof. Let the Hamiltonian be $\frac{1}{2} x^{\top} \mathrm{H} x$ and the vector field be $f=J^{-1} \mathrm{H} x$. The RK method yields $x^{\prime}=$ $R\left(h J^{-1} \mathrm{H}\right) x$,

$$
H\left(x^{\prime}\right)=\frac{1}{2} x^{\top} R\left(h J^{-1} \mathrm{H}\right)^{\top} \mathrm{H} R\left(h J^{-1} \mathrm{H}\right) x,
$$

and is thus energy-preserving iff $R\left(h J^{-1} \mathrm{H}\right)^{\top} \mathrm{H} R\left(h J^{-1} \mathrm{H}\right)=\mathrm{H}$ for all $h$ and for all symmetric matrices $\mathrm{H}$. We have $R\left(h J^{-1} \mathrm{H}\right)^{\top} \mathrm{H}=R\left(\left(h J^{-1} \mathrm{H}\right)^{\top}\right) \mathrm{H}=R\left(-h \mathrm{H} J^{-1}\right) \mathrm{H}=\mathrm{H} R\left(-h J^{-1} \mathrm{H}\right)$, which gives the result.

Note that self-adjointness and preservation of arbitrary quadratic invariants are sufficient but not necessary for $R(z) R(-z) \equiv 1$ to hold. The RK method with $b=\left(\frac{1}{3}, \frac{2}{3}\right)$ and $a=\left(0,0 ; \frac{1}{4}, \frac{1}{2}\right)$ has $R(z)=\frac{1+z / 2}{1-z / 2}$, hence $R(z) R(-z)=1$, but is not self-adjoint. Nor does it preserve arbitrary quadratic invariants. Preservation of quadratic energies provides $s$ conditions (of degree $2 s$ ) on the RK coefficients, self-adjointness and preservation of arbitrary quadratic invariants provide $s(s+1) / 2$ conditions (of degree 1 and 2 , respectively) each.

We do not know necessary and sufficient conditions for an RK method to preserve polynomial energies of a given degree $d$. We conjecture that for each $d$ and number of stages $s$, there is a $p(s, d)$ such that an RK method that is pseudo-energy-preserving up to degree $p$ is energy-preserving for polynomials of degree $\leq d$. For $d=2$ we have $p=2 s$. For cubics, consider 2 -stage methods. We find that there are methods that are pseudo-energypreserving of order 3 that are not energy-preserving, but that all methods that are pseudo-energy-preserving of order 4 are energy-preserving. These satisfy 5 conditions on their 6 parameters; there is a 1-parameter family of such methods, given by (10) for 2-node quadratures exact on quadratics. Thus $p(2,3)=4$.

Clearly no explicit RK method can be energy-preserving, even for (nonlinear) polynomial Hamiltonians. However, one can find explicit RK methods that are pseudo-energy-preserving to low orders. At orders 1 to 4 there are 1, 1, 1, and 3 conditions to preserve energy (and be consistent). The methods

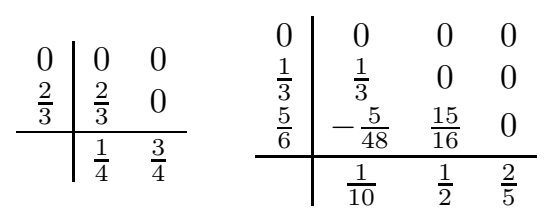

are the unique minimal-stage methods that are pseudo-energy-preserving of orders 3 and 4 but no higher.

Further results on energy-preserving integrators and the structure of B-series are contained in our forthcoming article [2].

Acknowledgements. W.M.W. acknowledges support of NZST and the Australian Research Council (ARC). R.I.M. acknowledges the support of the Marsden Fund of the Royal Society of New Zealand. G.R.W.Q. is the recipient of an ARC Professorial Fellowship. B.O. and G.R.W.Q. are grateful to the Lorentz Center, where some of the finishing touches to this paper were applied. 


\section{REFERENCES}

[1] M.-P. Calvo, A. Iserles and A. Zanna, Numerical solution of isospectral flows. Math. Comput. 66 (1997) 1461-1486.

[2] E. Celledoni, R.I. McLachlan, B. Owren and G.R.W. Quispel, Energy-preserving integrators and the structure of B-series. Preprint.

[3] P. Chartier, E. Faou and A. Murua, An algebraic approach to invariant preserving integrators: The case of quadratic and Hamiltonian invariants. Numer. Math. 103 (2006) 575-590.

[4] G.J. Cooper, Stability of Runge-Kutta methods for trajectory problems. IMA J. Numer. Anal. 7 (1987) 1-13.

[5] E. Faou, E. Hairer and T.-L. Pham, Energy conservation with non-symplectic methods: examples and counter-examples. BIT 44 (2004) 699-709.

[6] E. Hairer, C. Lubich and G. Wanner, Geometric Numerical Integration: Structure-Preserving Algorithms for Ordinary Differential Equations. Springer, Berlin, 2nd Edition (2006).

[7] A. Iserles and A. Zanna, Preserving algebraic invariants with Runge-Kutta methods. J. Comput. Appl. Math. 125 (2000) 69-81.

[8] R.I. McLachlan, G.R.W. Quispel and G.S. Turner, Numerical integrators that preserve symmetries and reversing symmetries. SIAM J. Numer. Anal. 35 (1998) 586-599.

[9] R.I. McLachlan, G.R.W. Quispel and N. Robidoux, Geometric integration using discrete gradients. Phil. Trans. Roy. Soc. A 357 (1999) 1021-1046.

[10] G.R.W. Quispel and D.I. McLaren, A new class of energy-preserving numerical integration methods. J. Phys. A 41 (2008) 045206 .

[11] J.E. Scully, A search for improved numerical integration methods using rooted trees and splitting. MSc Thesis, La Trobe University, Australia (2002).

[12] L.F. Shampine, Conservation laws and the numerical solution of ODEs. Comput. Math. Appl. 12B (1986) 1287-1296. 